\title{
THE PRESENT DAY TREATMENT OF GASTRIC AND DUODENAL ULCERS IN THE UNITED STATES.*
}

\author{
By ROLAND T. de HELLEBRANTH, M.D., (New Jersey, U.S.A.).
}

The old, almost feudal, controversy, which existed between physicians and surgeons concerning the method of treatment of gastro-duodenal ulcers for so many years, has been rapidly subsiding, and has given place to a very encouraging understanding. This armistice is due to several factors. Clinical observations and experiments have succeeded in convincing both parties that ulcers should not be considered as isolated local lesions, but as the outstanding and probably most painful symptom of metabolic disturbances which affects the patient physically and mentally. Therefore, the surgeon realises that excision of an ulcer or even the performance of gastric resection, without treating the patient's entire constitution, is similar to the treatment of a diabetic, gangrenous limb with ointments and lotions or even by amputation, and omitting the treatment of the patient's diabetes. The experiments of physiologists and close observations of the late results of operations, with the co-operation of internists, have proved to the surgeon, that there is no single operation that can be used for all cases of peptic ulcer and making use of the great selection of operative methods, naturally gave more permanent and startling results, lowering at the same time the mortality. This, in return, convinced physicians of the advantages of surgery and helped to eliminate much of the hesitancy and fear with which the internist would refer selected cases to the surgeon.

\section{SOME ATIOLOGICAL FACTORS.}

Without going into details in discussing the ætiology, we understand with Ochsner that the factors which cause gastro-duodenal ulcers are divided into two groups. In the first group are the so-called tissue-susceptibility and constitutional pre-disposition factors. These factors are not amenable, consequently the physician must concentrate on the hypersecretion, hyperacidity, and gastric trauma, which are the more controllable factors. Internists are in an advantageous position, because they have more opportunity to observe the patient as an individual, and even if they are not able to eliminate the factors of the first group, they can produce some ameliorating changes in them.

\section{INDICATIONS FOR OPERATION.}

An understanding between internists and surgeons has helped considerably in laying down the fundamental principles of indications for surgical intervention. Accordingly, operation is indicated in acute perforation, in cicatrized obstruction of the pylorus, in hour-glass deformity of the stomach, and in cases of chronic perforation or other involvement of neighbouring organs. Furthermore, we must seriously consider surgery as the only hope for the patient in those cases which stubbornly resist any and all kind of medical treatment, or which cause repeated massive hæmorrhages, and finally where the patient's social position does not permit him that prolonged rest and hospitalization which a successful conservative medical method would require. Surgery is also justified if the location of the ulcer and the patient's age are strongly suggestive of malignant changes which are either present already at the time the patient presents himself, or may occur in the near future. 
Briefly, with the exception of acute perforation, and cicatrized obstruction of $\frac{3}{0}$ the pylorus, medical treatment should be tried first in almost every case.

\section{THE TREATMENT OF HAMATEMESIS.}

What acute perforation is to the surgeon, the same is hæmorrhage from gastroduodenal ulcer to the physician. These hæmorrhages are very rarely fatal, and observations have shown that if they recur, they seldom come from the same ulcer. The bleeding patient is, naturally, put to bed immediately, and no nourishment is given by mouth. A hypodermic injection of 0.01 or 0.02 grams $\vec{\circ}$ of morphine hydrochloride and 0.001 gram of atropin sulphate, to be repeated every four hours, secures the necessary mental and physical rest, and the intravenous injection of ten cubic centimetres of Thromboplastin may lessen the severity of the bleeding. The physician can then direct his attention to the patient's general condition. If the loss of blood is not great, and the patient is not in a state of complete shock, with rapid, weak pulse, and cold, clammy skin, the intravenous administration of five hundred cubic centimetres of six per cent. Acacia solution is very beneficial, as this has been found to be the best substitute for blood. But since hæmorrhages recur so frequently within a few hours, the patient's blood should be grouped immediately and the necessary donor secured. If the loss of blood is considerable, two to five hundred cubic centimetres of blood should be given intravenously at the rate of ten to fifteen cubic centimetres per minute, and it should be repeated one to three times in the course of thirty-six hours. An ice bag is placed on the epigastrium, but the rest of the patient's bodf kept warm. A few authors tried the heroic method of applying gastric lavages with fairly warm solution of natrium hydrocarbonate or with a I:3000 solution of nitrate of silver. I saw a case of desperate hæmorrhage in Philadelphia, where everything failed and surgical operation was being considered, when strenuous gastric lavage promptly stopped the bleeding. On the other hand, I should hesitate to pour large amounts of water into the patient's stomach when one does not know how thin the base of the bleeding ulcer might be, or whether there are other ulcers which might easily perforate if the internal pressure is raised. The most suitable way of freeing the stomach of blood clots, gastric juices and eventually remnants of food is by means of a duodenal tube, which, inserted through the nose of the patient is kept in his stomach for 48 to 72 hours, making 3 it possible to cleanse his stomach without unnecessary trauma. After the first twenty-four hours, small amounts of ice-cream or ice-water can be given with a few sips of ice-water, and the third day a full puree diet, together with magnesium carbonate. The idea of starving the patient at a time when he is in special need of support, is now being abandoned chiefly because it cannot be considered to the patient's advantage if his stomach contains free acid and no food. The patient's anæmia is treated by generous doses of iron hypodermically, and by liver extract, given by intramuscular injections.

\section{THE MEDICAL TREATMENT OF GASTRIC AND DUODENAL ULCERS.}

As we all know, duodenal ulcers occur much more frequently than gastric ulcers, but since their treatment is practically the same, I shall not discuss it separately. The most popular method of treatment is that of the Mayo Clinic, of which I shall deal with in greater detail. It is a generally accepted idea that 0 ulcer patients should be treated just as strictly and thoroughly as tuberculous or $\frac{0}{D}$ diabetic patients. Their hospitalization is essential. It is only there that they? can be compelled to follow a rigid régime, and be prevented from following their $ळ$ 
usually irregular or hectic life, and be deprived of tobacco, coffee, or alcoholic beverages - in other words, it is in hospital where the patient can lead an altogether different life, and may expect quick relief or cure.

We must distinguish between mild or uncomplicated and grave or complicated cases, which are associated with great gastric retention. This complication, however, is due to inflammatory œdema of the pylorus and not to cicatrized obstruction. In both cases, the patients are put to bed and mild sedatives given to them. The most effective drug was found to be luminal of which threequarters of a grain given three times daily, suffices to help the patient relax and not look forward to the future with anxiety. Often this relaxation alone helps to decrease the production of abundant gastric acid. Smoking is forbidden. In our experience, tobacco in any form is most harmful to these patients, especially if it is used between meals when the stomach is empty, and hyperacidity greatly aggravates the condition. We agree with Friedrich who claims that nicotine not only produces spasm of the blood vessels but also increases gastric secretion. Most likely, the vasospasm is due to the increase in adrenalin resulting from the action of nicotine on the adrenal glands. The ischemia then results in necrosis.

\section{Uncomplicated Cases.}

In uncomplicated cases, the usual Sippy diet is followed with some modifications, which are mentioned briefly. The patient receives ninety cubic centimetres of a mixture of equal parts of milk and cream hourly from 7 a.m. until 9 p.m., and a powder is given four times daily which contains 0.65 grams of calcium carbonate, and 0.65 grams of heavy magnesium oxide. If the bowels become too loose, the heavy magnesium oxide is replaced by 0.65 grams of bismuth subcarbonate. The use of the popular natrium hydrocarbonate has been more or less discontinued, following the investigations of Lockwood and Chamberlain, as it was found that in order to neutralize gastric acidity for several hours, so much natrium hydrocarbonate was necessary as to produce alkalosis. If used in smaller doses, the acidity-after a very short time-not only returns but appears to be much higher than it was before the natrium hydrocarbonate was administered. Therefore, according to Loevenhart and Crandall, the use of calcium carbonate is more advisable, because "when suspended in water, it is neutral in reaction, but it also neutralizes the gastric acid to form calcium chloride and carbon dioxide, and finally, it can be given almost ad libitum. If taken in excess, it will coat over ulcerated areas, and may in this manner protect them from the action of irritants."

\section{Complicated Cases.}

In complicated cases with great gastric retention, instead of gastric lavage the duodenal tube is inserted, but only for the night. Great benefit is seen after the use of atropin, mostly in the form of tincture of Belladonna, given three or four times daily in ten drop doses. This not only decreases gastric secretion but also relaxes the pyloric sphincter muscle.

Since the aim in the therapy of gastro-duodenal ulcers is not only to decrease the production of gastric juices by eliminating irritating factors, but also to neutralize acidity, it was the idea of Winkelstein to administer milk and alkalies day and night through an indwelling gastric catheter. He suggests a mixture of five grams of natrium hydrocarbonate and one litre of milk and, with the aid of a Murphy-drip, to be given through the duodenal tube at the rate of 30 drops 
per minute. This method will require about 3,000 c.c. of milk and I5 grams of natrium hydrocarbonate in twenty-four hours. In this form natrium hydrocarbonate is much more effective than when given only four or five times daily, although it can be substituted by the same amount of magnesium carbonate. This treatment can be continued for ten to fourteen days and although it may inconvenience the patient, especially at the beginning and may necessitate the administration of sedatives, the result is so startling and the relief obtained so immediate that I have seen patients securing the duodenal tube themselves and inserting it at their home as soon as their ulcer symptoms appeared. The very discomforting dryness of the throat is easily relieved by giving small sips of water or olive oil by mouth occasionally.

Often good results have been seen in the neutralization of gastric juices by means of mucin as suggested by Fogelson. He recommended its use mostly in patients with intractable ulcer symptoms, and with Hurst he claims that "the $\vec{N}$ mucous mechanically blocks the mouths of the secreting tubules and combines with some of the free acid. It is not unlikely that ulcer-bearing patients lack adequate amounts of mucous and the administration of mucin gives relief and facilitates healing." The best way to give it, is in 30 to 60 c.c. doses three times daily, dissolved in warm water or chocolate milk mixtures, and in severe cases in capsule form, containing 2 grams of mucin, every hour, between meals. This drug, however, is too recent to be recommended as an absolutely reliable remedy, although in combination with acid-free feedings it often proves to be extremely valuable.

On Winkelstein's principle Woldman and Rowland successfully control gastriẹ acidity by continuous aluminium hydroxide drip. A small duodenal tube, size I2, is passed through the patient's nostril into his stomach. 200 c.c. of $7 \%$ colloidal aluminium hydroxide is added to 600 c.c. of fresh distilled water, and stirred until well mixed and poured into a bottle from which, with the aid of a Murphy drip, the solution will flow into the stomach at the rate of 5 or 6 drops per minute. The aluminium hydroxide drip is free from the danger of alkalosis, and can be given in addition to the milk and cream feedings.

In average cases, the patient is kept in bed for three weeks. After the first week, when he is free from actual pain, the administration of alkalies and the hourly feedings are continued, but three or four times the milk and cream mixture is replaced by cream soups, made of celery, asparagus, or green peas. Also, cooked cereals with cream are given until the end of the second week, when vegetable purees may be permitted, and cooked fruits. The intervals between two feedings may be increased to an hour and a half, later to two hours, but never longer. The alkalies and tincture of Belladonna are also given together with luminal. On the fourth week, other vegetables are permitted, also the white meat of chicken, preferably minced, and a few slices of bread with just enough butter to make it palatable. Fruit jellies, custards, puddings, are added to the diet, but no spices other than a moderate amount of salt are allowed. Coffee is strictly forbidden, because it not only prevents the patient from relaxation, but also increases gastric secretion.

In cases of pyloric obstruction caused by spasm or œdema, " the condition may be corrected with the same intensive treatment with the addition of gastric lavages, which are done morning and night, and at the end of the second week an 
ordinary meal is given, consisting of potatoes, meat, one cooked vegetable, tea, toast, butter, half an orange, and six or eight seedless raisins. Seven hours later the content of the stomach is aspirated. If the evidence of gross retention exists, the obstruction is most likely due to organic changes and should be corrected by operation."

\section{The Use of Certain Drugs.}

In the last few years extensive experiments were started with different parenteral methods. The results reported vary greatly, and show that the real cure for gastro-duodenal ulcers has not yet been discovered. The most popular drugs are histidine hydrochloride, in the form of larostidine, and insulin with or without the combination of pepsin.

Histidine. The use of histidine is based upon the theory that peptic ulcer is " due possibly to a lack of some material, essential to the resistance and normal repair of tissues, especially that of the gastric and duodenal mucosa. By deflecting the natural path of the duodenal fluid, Mann and Williamson were able to produce ulcerous lesions in the intestinal tract of dogs, together with other symptoms, indicative of severe metabolic dysfunction. The administration of histidine produced quick reconstruction in the mucosa and helped the ulcerous lesions to disappear." Those who so fervently advocate the use of larostidine must admit that it is not the ideal cure for peptic ulcer-bearing patients because only very small, almost insignificant lesions responded so promptly to the treatment and often the symptoms reappeared as soon as treatment was discontinued. The drug and method have undoubtedly a well deserved place in the treatment of peptic ulcers but only in combination with acid-free diet and proper rest. Its use is, however, not compatible with very rigid dietary rules because according to the theory of those who made extensive experiments with histidine, ulcerbearing patients are unable to synthetize amino-acids and histidine among them, so that they must obtain them from the food they consume. Consequently strict diet would only cause a vicious circle. On the other hand it is undeniable that certain food plays an important rôle in the ætiology of ulcers, for example, I understand, an unusually high number of Abyssinians are suffering with peptic ulcers and they all consume large amounts of cayenne pepper.

Insulin. The insulin treatment is unscientific, yet Danzer reports good results with it. He mixes 15 drops of insulin, taking the U.20 solution, with half a teaspoonful of bismuth subcarbonate in two ounces of water, which is allowed to stand for 30 minutes and the patients swallow it slowly half an hour before each meal. Two tablets of yeast are taken with a little water three times daily after each meal. Every fourth day one to one and a half c.c. of distilled water is injected intravenously and the body is irradiated with ultra-violet light, probably with the aim of activating the vitamin $B$. contents of the yeast.

At the Mayo Clinic, duodenal and jejunal feedings are carried out, mostly following jejunostomy only, although some authors recommend its use even for treating severe gastritis. A fine Sawyer tube is inserted through the nose, down into the jejunum, for a distance of IOO to I2O centimetres. Intelligent patients learn to do it themselves. The nourishment then consists of butter, eggs, peptonized milk, flour, sugar, and fruit juices.

Repeated roentgenological examinations are made, of course, and the disappearance of the niche is the aim of the different treatments. We must not 
forget, however, that the ulcer is not always completely healed when it does not show in the X-ray picture. It may be filled out by débris, or food particles, or by weak, fresh granulation tissue which is soon destroyed, and the patient soon relapses. The patients are on the strictest diet, and are told to follow the most rigid régime for six months. After that, slight modifications are made, but the patient is never allowed to smoke, to drink coffee or alcoholic beverages, or use condiments with his food. He should not allow more than two and a half to three hours to elapse between meals, so as to prevent the accumulation of gastric secretion.

An important question is the regulation of the bowels. Ulcer-bearing patients usually suffer with constipation. The different cathartics would only stir up the irritable colon and create more gas. Therefore, especially at the beginning of treatment, two tablespoonfuls of liquid Petrolatum is recommended at night, and a soap-suds enema in the morning if necessary. In most cases, however, the magnesium oxide regulates the bowels.

\section{The Danger of Alkalosis.}

The danger of alkalosis mostly threatens patients suffering from renal and hepatic diseases, arteriosclerosis, or arterial hypertension. In these cases, usually at the end of the second week of intensive treatment, the patient will complain of " sour taste in his mouth, a sudden distaste for milk, later of dizziness, pains in the joints, nausea, general weakness. In severe cases, mental apathy, slowing of respiration, drowsiness, and even convulsions may develop. To relieve these symptoms, the number of feedings should be decreased and the alkalies stoppedo temporarily at least," and substituted by Kaolin or mucin. Kaolin is one of the most useful substances in substituting alkalies, especially in combination with aluminium hydroxide and liquid petrolatum. This is given three times daily, before meals, in a little water. Immediately fresh fruit juices should be added to the diet, and operation considered, providing the patient's general condition permits it.

\section{THE SURGICAL TREATMENT OF GASTRIC AND DUODENAL ULCERS.}

\section{(a).-Perforation of the Ulcer.}

The most important indication for immediate surgical intervention is acute perforation of gastro-duodenal ulcer. Whether we should operate, even if the critical first thirty hours have passed already, will depend on the patient's physical condition. Emaciated, dehydrated patients with impaired renal or hepatic function have a better chance to recover if, after the first thirty hours, only supporting and conservative measures are taken. Well developed and otherwise organically healthy individuals on the other hand may easily recover, even if the operation is performed forty to forty-eight hours after the perforation occurred. The principle to follow in these cases is to open the abdomen and get out of it as quickly as possible. Suturing the ulcer and attaching part of the omentum to it or merely plugging the perforation with a small piece of omentum is usually the best method, followed by the insertion of several cigarette drains. I do not suggest the performance of a gastro-enterostomy, because it only prolongs the operation, and the insertion of a duodenal tube through the patient's nostril will drain off the gastric contents equally well. 


\section{(b).-Chronic Ulcer.}

Concerning the operative management of chronic peptic ulcers, there is still controversy between surgeons who advise conservative methods and those who favour radical operations. The Mayo Clinic exercises more conservatism with excellent results, on the other hand Berg, Blahd and others who perform gastric resection almost as a routine are able to report equal success. I believe that the combination of both methods is the ideal. No surgeon should insist on performing gastric resection in an old emaciated patient with complete pyloric obstruction, where a simple gastro-enterostomy will be life-saving and will give him comfort and relieve him of his symptoms. On the other hand, excision with or without the combination of gastro-enterostomy or pyloroplasty will not give the desired permanent result in a patient who is suffering with a deep, penetrating crater, large acid producing surface and no pyloric obstruction. The surgeon should select his operative method just as carefully as he selects his operative cases.

(i) Excision of the Ulcer. Excision of the ulcer is a justified method only if the lesion is discovered in the course of other operations, for instance, on the biliary tract. A small, almost healed ulcer, in the duodenum, or the lesser curvature, without involving the pylorus should be excised either by cautery or by knife. The cautery gives better results as regards healing. In these cases excision is the operation of choice, the purpose being to relieve the patient of his ulcer symptoms. It should always be combined either with pyloroplasty or gastroenterostomy. After all, the aim of the operation is not only to remove the lesion temporarily, but also to change the motor and secretory function of the stomach permanently, and if no adequate drainage is offered, recurrence will be almost certain.

Wedge-shaped excision of ulcers alone on the lesser curvature is strictly contraindicated, because as Alvarez proved it, it has a deleterious effect on gastric peristalsis.

(ii) Pyloroplasty. Concerning pyloroplasty, we agree with Ochsner, who says " granted that the pyloric disfunction is either the relaxation or spasm of the muscle, theoretically the most physiological procedure would be excision of the pyloric sphincter, as suggested by Judd, Deaver, and Burden. The operation consists of excision of a portion of the pyloric sphincter which will permit emptying of the stomach without retention and also a ready regurgitation of the alkaline duodenal secretion into the stomach in order that neutralization may occur. Judd and Waldron emphasized the importance of excision of from two-third to three-fourth of the pyloric sphincter because if less of the muscle is removed, recurrence of the symptoms occur." Judd practically converts the stomach and duodenum into one continuous tract, thereby producing almost the same physiologic changes that occur in cases in which gastro-enterostomy has been performed. The mortality rate is less than $\frac{1}{2} \%$ and is a suitable operation for younger people.

(iii) Cholecysto-gastrostomy. Based on similar principles, to neutralize gastric acid by duodenal secretion or bile-some authors recommended cholecystogastrostomy. A few months ago I operated on a fifty-four year old woman who was suffering with grave jaundice and severe digestive disturbances. The jaundice was considered to be due to carcinoma of the head of the pancreas and 
operation revealed a carcinoma of the pancreas, but also a duodenal ulcer of considerable size. Considering her outlook on life and health, and her general condition, I resorted to cholecysto-gastrostomy and she made an uneventful recovery from the operation. I was surprised to find that her digestive disturbances, probably due to the presence of the duodenal ulcer, have completely disappeared. Roentgenological examination showed no evidence of the duodenal lesion and the patient seemed to gain in strength and weight.

(iv) Gastro-duodenostomy. Gastro-duodenostomy is a method that deserves some consideration in the treatment of duodenal ulcers, although it is necessary that no extensive adhesions are present preventing the mobilization of the duodenum and stomach. This operation is simple and easy to perform and is quite logical from a physiological point of view, in as much as the stomach continues to empty into the duodenum, but avoids the ulcer, which should be sutured over with a strong silk suture, completing the obstruction of the pylorus.

(v) Gastro-enterostomy. Gastro-enterostomy-a favourite operation of the Mayo Clinic with and without combination of other methods is losing its popularity, because of the complication of jejunal ulcer. As Matthews and Dragshed showed "the susceptibility of the intestinal tract to acid gastric chyme increases proportionately with the aboral distance from the pylorus." And as Ochsner says: "in an individual with ulcer diathesis the performance of a gastroenterostomy is not without danger, because it permits the acid gastric chyme to be emptied into the jejunum, which is less resistent to the digestive action of the gastric juice than the gastric or duodenal mucosa."

The occurrence of jejunal ulcers, following gastro-enterostomy, is between $5 \%$ and $30 \%$, according to different authors, and even if we are more sceptical about the higher figures, we must admit that Blahd is right when he claims, that if gastro-enterostomy with or without excision of the ulcer were the ideal method, then surgeons would be satisfied with it and perform it routinely without making the greatest effort to find a more suitable operative procedure. Recurrences are fairly frequent after gastro-enterostomy, but we must admit, that it has been performed very often indiscriminately, and even in the absence of peptic ulcer. It must never be performed with a patent pylorus, which should be occluded either by the cicatrizing ulcer or in the course of the operation. The main objection to gastro-enterostomy alone is that it does not remove the lesion itself. This is not so important in cases of duodenal ulcer, but in the case of gastric ulcer, the danger of malignant changes, present at the time of the operation or developing in the near future is so great that practically all methods should also aim at the removal of the lesion.

(vi) Gastrectomy. The danger of malignancy developing in gastric ulcer is one of the most logical arguments of those who fervently advocate gastric resection as the operation of choice. In addition they very rightly claim that gastrectomy fulfils other important requirements, such as decreasing the acid producing surface and also preventing recurrence. Not that there is no danger of recurrence following gastrectomy, but they are less frequent and some surgeons claim $90 \%$ cure. In the hands of a well-trained gastric surgeon, the mortality after gastric resection is just as low as following simple gastro-enterostomy. Even the Mayo Clinic advocates it in "all large lesions if it can be carried out, without undue risk, especially where there have been severe and repeated hæmorrhages and where previous more conservative operations were followed by recurrences.' 
(vii) Division of the Stomach and Gastro-enterostomy. There are patients suffering with extensive or multiple ulcers of the duodenum where gastric resection cannot be performed. Pyloric occlusion does not give good results combined with gastro-enterostomy, because the large, acid producing surface of the stomach remains undiminished and the appearance of a jejunal ulcer is almost a certainty. In these cases an operation was found to be advisable, which simply divides the stomach at a sufficiently high level in order to reduce acidity leaving the lesion untouched and finished by a gastro-enterostomy. This operation has the distinct advantage over gastric resection in that it is less dangerous and at the same time accomplishes more than gastro-enterostomy because the lesion is completely protected from further irritation and there is the possibility of permanent healing of the ulcer. If a recurrence necessitates the performance of a more radical operation later on, that can always be carried out without any difficulty.

(viii) Excision of Ulcer and Pyloroplasty. Great popularity is enjoyed by excision of duodenal ulcers, combined with pyloroplasty. This method removes the lesion, which is a distinct advantage in cases of bleeding ulcers, and also permits a thorough digital investigation of the posterior wall where multiple duodenal ulcers are easily overlooked. In order to perform this operation, it is essential that no extensive scarring should be present in the duodenum, and the ulcer must not be too large on the posterior wall, or perforating into the pancreas, or involve the liver or gall-bladder.

\section{Anzmia after Gastrectomy.}

The anæmia which occasionally develops after gastric resection cannot be brought up as a justified contra-indication to this operation, because with the administration of liver extract and large doses of iron, it can easily be controlled. I suggest the administration of 5 c.c of liver extract intramuscularly for three consecutive days following operation. Then one injection of the same amount of liver extract should be given weekly for two months. Finally one injection given monthly almost indefinitely. Of the iron products, "ferric ammonium citrate is preferable, because it contains more metallic iron than the Blaud's pill and does not cause constipation. Usually 4 to 6 grams are given in gelatine capsules or as Monot suggested a simple $50 \%$ aqueous solution of iron and ammonium citrate can be prescribed in oral doses of 2 to 6 grams daily."

\section{THE GENERAL METABOLISM OF THE PATIENT.}

Attention has been called already to the main factors in the ætiology of peptic ulcer, namely, the metabolic disturbances, and these are not amenable to surgical treatment. G. W. Crile in Cleveland tried to overcome this difficulty by the operation of denervation of the adrenal glands. In his opinion "the underlying cause of ulcer is, in many respects, the same as that of exophthalmic goitre or neurocirculatory asthenic-states." He describes several cases, in which the most radical gastric surgery failed to cure the patient and constant recurrence could only be prevented by denervation of the adrenal glands. It is a difficult operation in many respects, and it is thought that with some modification, and in wellselected cases this method has a very promising future. But whether the treatment is a surgical or medical one, we must never overlook focal infection. Thorough investigations in large series of cases, have revealed the same nonhæmolytic streptococci, especially at the edge of the ulcer, which might have come 
from teeth, tonsils, and diseased abdominal organs such as the appendix or gallbladder. Non-removal of these foci of infection might easily render futile the best treatment.

\section{SUMMARY.}

Summarizing my remarks, I will repeat that the first principle to follow in the course of the treatment of peptic ulcer is to recognize it as a symptom of a general metabolic disturbance and not to rely only on either medical or surgical methods to produce the cure of the lesion. Physicians should also endeavour to assess the chances the patient may have of being cured by conservative methods, and should have less fear of referring them to the surgeon before all available drugs and methods have been tried out and the patient has become emaciated, and developed malignant changes. On the other hand, surgeons, irrespective of good results obtained by operation should not stop there and believe that their work is finished and the patient permanently cured, but should refer him back to the physician whose constant care will help greatly in preventing recurrence.

\section{REFERENCES.}

Enslerman and Balfour: The Stomach and Duodenum-(Saunders, 1936).

Ochsner: Peptic Ulcer. Surg. Gyn. and Obst., 1936.

Danzer: The Insulin Treatment of Peptic Ulcer. Medical Record, 1935.

Behneman: Late concepts of Peptic Ulcer, and a preliminary report of Modern Therapy. Northwest Medicine, 1935

Miller: A present-day review of Gastric and Duodenal Ulcer. The New England Journal of Medicins. 1932.

Hund-Verne: The Surgical Treatment of Peptic Ulcer. Southwestern Medicine, 1935.

Blahd: Gastrectomy. Surg. Gyn. Obst., 1936.

Blahd: Gastrectomy. Surg. Gyn. Onst., 190.

Winkelstein: The continuous milk-administration in Peptic Ulcer-therapy. Ann. Jour. of Med. Sc., 1933. Abell: The Diagnosis and Treatment of Duodenal Ulcer. Southern Medical Journal, 1935.

Fogelson: Gastric Mucin Treatment for Peptic Ulcer. Archives of Internal Medicine. 1935.

Judd and Phillips: Pyloroplasty: Its place in the Treatment of Peptic Ulcer. Annals of Surgery, 1934.

Heuer:- The Choice of Operations in the Treatment of Peptic Ulcer. New York State Journal of

Heuer: The Chedicine, 1935 .

Felter : and Weintraub: The Results of Medical and Surgical Treatment of Peptic Ulcer. Annals of Surgery, 1933.

Burke : The Operative Mortality and Morbidity of Partial Gastrectomy for Peptic Ulcer. Surgery, Gynæcology and Obstetrics, 1931.

Eusterman: Gastric and Duodenal Ulcers; Factors Essential to their successful Medical Management. The Wisconsin Medical Journal, 1935.

Abell : The Surgical Treatment of Peptic Ulcer. The West Virginia Medical Journal, 1931.

Hinton: The Management of Patients with Gastric and Duodenal Ulcers. Annals of Surgery, 1931.

Underwood: The Use of Mucin in the Treatment of Intractable Peptic Ulcer. Texas State Journal of Medicine, 1934. 